\title{
Potential of surfactant as anti-shear additive for DRA of oil
}

\author{
Xiaodong Dai, a , Guoping $\mathrm{Li}^{2}$, Jingmiao $\mathrm{Li}^{2}$, Yanping Xin ${ }^{1}$, Guanghui Yang ${ }^{1}$, \\ Yanzong Zhang ${ }^{3}$ \\ ${ }^{1}$ Shengli college China University of Petroleum, Dongying, Shandong 257061, China \\ 2 PetroChina Pipeline Company, Langfang, Hebei 065000, China \\ ${ }^{3}$ Sichuan Agricultural University, Chengdu, Sichuan 611130, China \\ axiaodongdai1980@163.com
}

Keywords: surfactant, drag reduction, anti-shear.

\begin{abstract}
Poly-olefin as drag reduce agent lost most of drag reduction capacity by shear degradation. The surfactant also was used as DRA, known to possess drag reduction and shear stability as formed reversible micelles. Combining poly -olefin and surfactant, the potential of surfactant as DRA anti-shear additives were investigated in research. Four DRA samples with surfactant were prepared, drag reduction and anti-shear performance were analyzed through experimental loop. It was shown adding surfactant could reinforce the drag reduction, caused by surfactant micelle drag reduction effect. Simultaneously, surfactant could also improve shear stability of Poly-olefin, No.5 sample left $40 \%$ drag reduction capacity. This could be explained that poly-olefin and surfactant formed microstructure by interaction, surfactant micelles could protect poly -olefin through reversible structure, and shown shear resistance. Surfactant could be a potential anti-shear additive for DRA of oil.
\end{abstract}

\section{Introduction}

The effect of drag reduction allows significantly for the increase of the flow rate without the necessity of increasing power consumption. Drag reduce agent (DRA) is a kind of chemical additive used in oil transportation, heat engineering, firefighting et al, for energy saving, throughput increase, and pipeline safety. Poly -olefin as DRA adding for crude oil pipeline was effective and had become one of the transportation technologies in real operation ${ }^{[1]}$. The extent of drag reduction increased with the molecular weight of the polymer increases. While, the poly -olefin with higher and more concentrated molecular weight would lost most of drag reducing rate by shear degradation. Commonly, DRA was injected station by station to maintain the drag reduction performance ${ }^{[1-4]}$. How to improve the shear stability had been investigated for many researchers.

Surfactants had been used as DRA of crude oil firstly, since they could form micelles. The formed micelles with associating superstructures could break reversibly after high shear stresses, which shown high drag reduction and shear stability. While surfactants as DRA had not been applied after polymer DRA invented, because they were effective at high concentrations, and sensitive to ions and temperature. Matraset al [5] have attempted to report the experimental examination of adding surfactant to polymer (PEO) as DRA, and shown positive results. The mixed polymer-surfactant aggregates presented better drag reduction and shear stability than pure polymer and surfactant, by their synergistic effect. The mixed PEO with surfactant was not used for crude oil. So, inspired by that, we did research about surfactant additive to poly -olefin for crude oil, and confirm the intensification of drag reduction and shear stability. Some kinds of surfactant and their mixer were explored and compared. 


\section{Experimental}

\subsection{Preparation of the surfactant additives}

Non-ionic poly -olefin with molecular weight of $>600$ millilon was the effective component of DRA slurry. A: Cationic surfactant cetyltrimetyl am. monium bromide (CTAB), salt sodium salicylat (as counter-ion), B: Amphion surfactant twelve alkyl two ethanol amide $\left(\mathrm{C}_{16} \mathrm{H}_{33} \mathrm{NO}_{3}\right)$, and C: Poly sorbitol (Tween-80) were used as surfactants. Firstly, each surfactant was added in DRA to evaluate their performance, and then, a mixed surfactant was tested. The designation and the composition of surfactant additives are presented in Table 1.

\begin{tabular}{cc}
\hline Solution designation & Solution composition \\
\hline No.1 & 25ppm poly -olefin slurry \\
No.2 & $10 \mathrm{ppm} \mathrm{A,} \mathrm{5ppm} \mathrm{NaSal}$ \\
No.3 & $10 \mathrm{ppm} \mathrm{B}$ \\
No.4 & $10 \mathrm{ppm} \mathrm{C}$ \\
No.5 & $5 \mathrm{ppm} \mathrm{A,} \mathrm{2.5ppmNaSal,} \mathrm{5ppm} \mathrm{B,} \mathrm{5ppm} \mathrm{C}$ \\
\hline
\end{tabular}

Poly-olefins particles was slowly added into surfactant solutions, and slurry were slowly stirred at $25^{\circ} \mathrm{C}$ for $2 \mathrm{~h}$, getting mixed DRA with additive. DRA was firstly dissolved in diesel at $25 \mathrm{ppm}$ for evaluation.

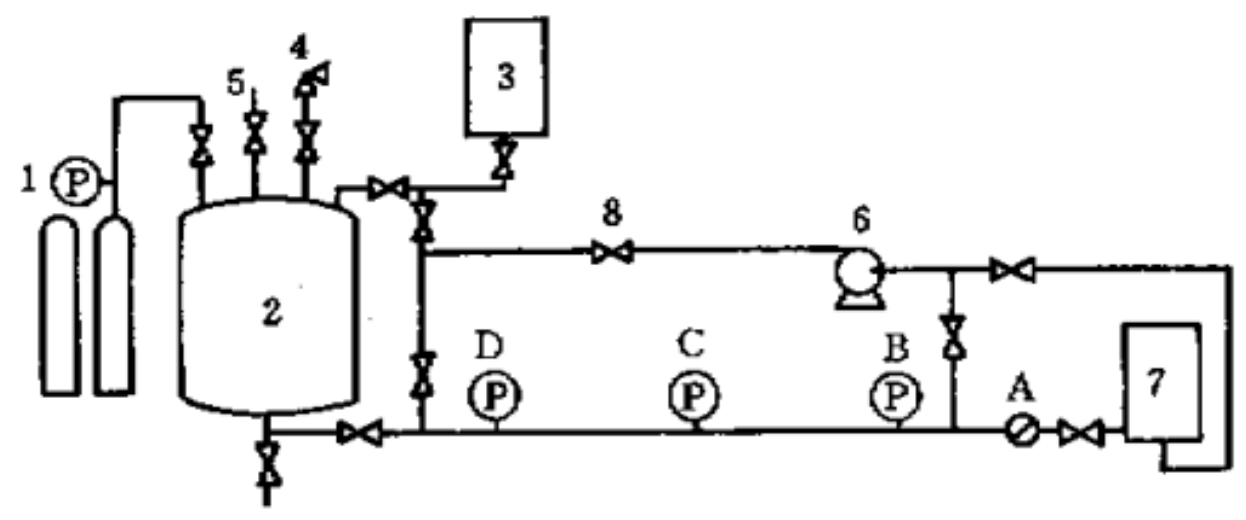

Fig.1 The apparatus for the performance evaluation of DRA

1-N2; 2-pressure surge tank; 3-dilution tank; 4-safety relief valve; 5-air relief valve; 6-grear pump; 7-reflux accumulator; 8-ball valve; A-flow sensor; B,C,D-pressure sensors.

\subsection{Drag reducing evaluation.}

The apparatus for the performance evaluation of DRA was shown in Figure 1. The drag reducing rate was affected by flow parameters, such as Reynolds number, so diesel was selected as evaluated fluid. Evaluation procedure (based on CNPC company standard) is as follows:

(1) Measuring the flow rate before (Q0) and after (Q1) addition of DRA;

(2) Calculating the flow increase rate: $\mathrm{TI}=(\mathrm{Q} 1-\mathrm{Q} 0) / \mathrm{Q} 0 \times 100 \%$

(3) Calculating the drag reducing rate: $\mathrm{DR}=[1-1 /(1+\mathrm{TI}) 1.79856] \times 100 \%$

(4) Investigating the anti-shear performance: The DR results from the no-sheared and sheared DRA, and DRA with additives were plotted, and the anti-shear performance was evaluated according to the tendency and slope of the curve.

\section{Results and Discussion}

\subsection{Drag reduction influence by adding surfactant}

The influence of different surfactant additives to drag reducing performance was investigated. Firstly, four kinds of surfactant additives was adding to poly -olefin slurry with setting ratio as shown in Table 1. Figure 2 shown the drag reduction results of five samples. It was observed that 
DRA without additive had 33\% reduction rate in diesel. While, after adding surfactant, drag reduction effect has different intensification ratio, it could be the micelle effect by surfactant. Surfactant would aggregated micelle in solution, and show drag reduction ability. No.5 sample mixed with A, B, C and NaSal presented the best drag reducing performance. No.1 sample, mixed cationic CTAB and counter-ion NaSal had less improvement. While, No.2 sample of amphion surfactant possessed the worst improvement to drag reduction, since it was hard to form micelles by polarity in a non-ionic surrounding circumstance. So, drag reduction capacity could be improved after adding surfactant by micelle drag reduction effect, it possessed.

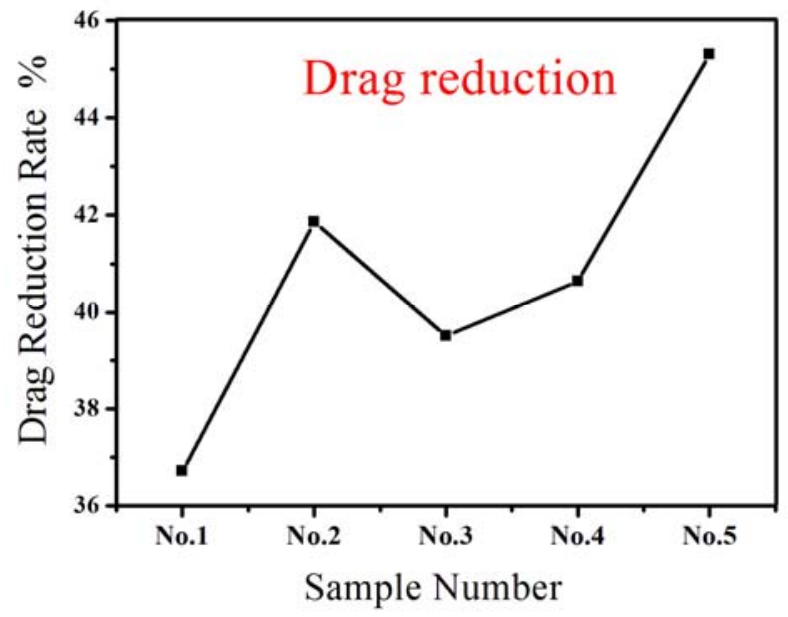

Fig.2 Influence of surfactant additive to drag reduction of DRA.

\subsection{Anti-Shear performance}

The DRA anti-shear experiments were carried out by drag reduction instrument. Solution with DRA was sheared in centrifugal pump, and the shear stability was analyzed by left drag reduction rate after shear. So, the anti-shearing results were shown in Figure 3.

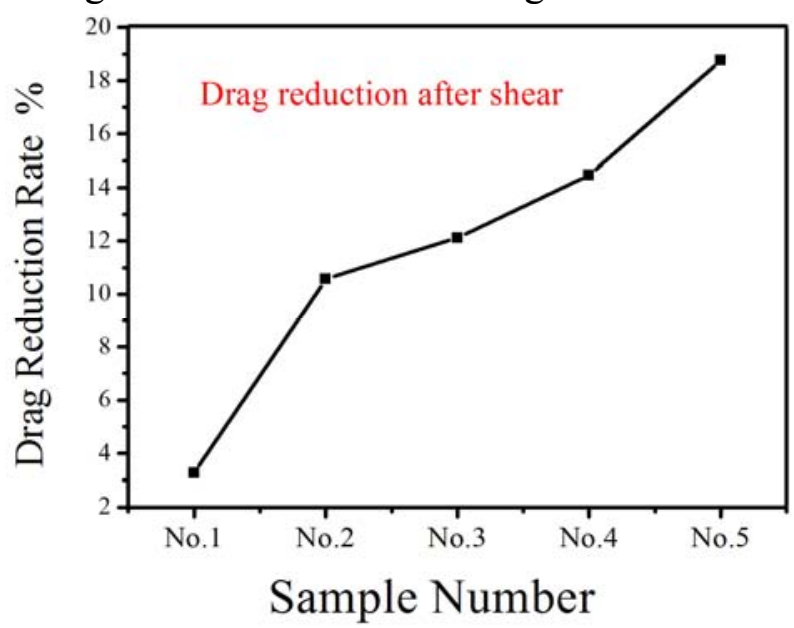

Fig.3Anti-shear performance of the DRA with surfactant additive.

After being sheared in centrifugal pump for first time, the drag reduction rate deceased more than $90 \%$, only $3 \%$ drag reduction rate left for No.1 sample without additive. That mean poly -olefin had no anti-shear capacity. After adding surfactants, shear stability of sample had great improvement for four samples. Especially, No.5 sample had left more than $40 \%$ drag reduction capacity with drag reduction rate of $18.75 \%$. This phenomenon of shear stability can be explained by a more stable configuration of the aggregate structure, where the micelles arranged in aggregates and the poly -olefin were entangled around micelles. During shear condition, poly-olefin undergone mechanical degradation. While, microstructure formed by poly-olefin and surfactant micelles shown shear resistance caused by polymer-micellar rebuilding by surfactant [5-8]. It meant surfactant could provide protection to polymer during shear conditions. So, it can be concluded from our studies that 
surfactant as shear stability additive could be a feasible method for DRA anti-shear performance, and it had shown great potential for further study.

\section{Conclusions}

(1) Poly -olefin DRA has no shear stability, while surfactant has shear stability by micellar rebuilding.

(2) Surfactant as additive to Poly -olefin DRA had shown improvements not only for drag reduction capacity, but also shear stability. The drag reduction rate left more than $40 \%$ after shear by adding surfactant.

(3) Poly -olefin and surfactant formed microstructure by interaction which could rebuild again, and shown shear resistance. It could be a potential direction for DRA development.

\section{References}

[1] Guan Zhongyuan, Li Chunman. Research and Application of EP Drag Reducer. Oil \& Gas Storage and Transportation, Vol.20(2001)No.8, p.32 34.

[2] Shi Yulin, Li Huiping, Zhou Qixiong,et al.Synthesis of Drag Reducer for Crude Oil from -olefin over TiCl4/Al(i-Bu)3 . Petrochemical technology, Vol.35(2006)No.4, p.371 375.

[3] Li Guoping, Li chunman, Zhang Zhiheng et al. Pipeline Chemical Additive Technology [M]. Petroleum Industry Press, 2010, p.12 20.

[4] Malik S., Shintre S. N., Mashelkar R. A. Enhancing the shear stability in drag-reduction polymers through molecular associations. Macromolecules, Vol.26 (1993), p.55-59.

[5] Matras Z., Malcher T, Gzyl-Malcher B., The influence of polymer-surfactant aggregates on drag reduction. Thin solids films, Vol.516 (2008), p.8848-8851.

[6] Mohsenipour A. A., Pal R., The role of surfactants in mechanical degradation of drag-reducing polymers. Industrial engineering chemical research, Vol.52 (2013)No.3, p.1291-1302.

[7] Mohsenipour A. A., Pal R., Synergistic effects of cationic surfactant and nonionic polymer additives on drag reduction. Chemical engineering communication, Vol.200 (2013)No.7, p.935-958.

[8] Matras Z., Kopiczak B., Intensification of drag reduction effect by simultaneous addition of surfactant and high molecular polymer into the solvent. Chemical engineering research and design, Vol.96 (2015), p.35-42. 